\title{
MEDICINA NARRATIVA: EL PACIENTE COMO “TEXTO”, OBJETO Y SUJETO DE LA COMPASIÓN
}

\author{
Carlos Alberto Rosas Jiménez ${ }^{1}$
}

Resumen: Las narraciones han llegado a influir en la medicina, dando lugar a un nuevo enfoque llamado "medicina narrativa". En este trabajo consideramos al paciente como un texto, es decir, un libro abierto que el médico interviene, pero también del que el médico puede y necesita mucho aprender. Ahondar un poco en la perspectiva narrativa de la comprensión del paciente y de su situación nos ayuda a descubrir cómo el paciente es objeto de la compasión de los médicos, pero también cómo se convierte en sujeto de esa compasión. Concluimos que es importante que los médicos dediquen tiempo en su formación médica inicial, así como en su formación permanente, a explorar y explotar el valor de las narraciones, que les ayuden a categorizar sus experiencias vividas y las de los pacientes.

Palabras clave: compasión, bioética, narrativa, paciente, texto

\section{Narrative medicine. The patient as "text", object and subject of compassion}

\begin{abstract}
Narrations have been able to influence medicine, giving rise to a new approach call "narrative medicine". In this paper we consider the patient as a text, such is, an open book that the physician intervenes, but also from which the physician may and need to learn a lot. To deepen a little in the narrative perspective of patient understanding and his/her situation helps us to discover how the patient is object of compassion by physicians, but also how he/she turns into subject of compassion. We conclude that it is important for physicians to dedicate time in their initial medical training, as well as in their permanent training, to explore and exploit the value of narrations, that help them to categorize their living experiences and that of the patients.
\end{abstract}

Key words: compassion, bioethics, narrative, patient, text

\section{Medicina narrativa: o paciente como “texto", objeto e sujeito de compaixáo}

Resumo: As narrativas estão chegando a influenciar na medicina, dando origem a uma nova abordagem chamada "medicina narrativa". Neste trabalho consideramos o paciente como um texto, em outras palavras, um livro aberto que o médico intervém, mas também, que deve aprender muito. Aprofundar-se um pouco na perspectiva narrativa da compreensão do paciente e de sua situaçáo ajuda a descobrir como o paciente é objeto da compaixão dos médicos, embora também como se converte em sujeito desta compaixão. Concluímos que é importante que os médicos dediquem mais tempo em sua formação médica inicial, bem como em sua formação permanente, para explorar o valor das narrativas que ajudam a categorizar suas experiências e a dos pacientes.

Palavras-chave: compaixão, bioética, narrativa, paciente, texto

\footnotetext{
${ }^{1}$ Grupo de Investigación KHEIRON Bioética, Universidad de la Sabana, Bogotá, Colombia

Correspondencia: carlosalbertorosasj@gmail.com
} 


\section{Introducción}

Hoy en día, como mencionan Miles y Loughlin y Miles y Mezzich(1), muchos pacientes experimentan un tratamiento impersonal y deshumanizante, tanto así que este fenómeno se presenta como una crisis dentro de los sistemas del cuidado de la salud. En esta realidad, a menudo se hace sentir a los pacientes como "el riñón en el cuarto 5 " o "el hígado en el cuarto 10", en lugar de personas con una historia única de enfermedad(1). Por tanto, la búsqueda de un mayor respeto a la dignidad humana sigue siendo hoy una tarea imprescindible para la humanización de la salud.

La bioética personalista y la medicina han arrojado nuevas luces que permiten abordar por diferentes flancos dicha problemática. Recientemente se han abierto nuevas ventanas que permiten la entrada de destellos de luz provenientes de diversas maneras de aproximarse a la persona humana. Entre aquello que se muestra como novedoso está el elemento narrativo, que ha sido explorado desde la filosofía hermenéutica de Ricoeur y por la medicina narrativa. En efecto, como dice Alzate: "en la narración hay posibilidad de conocimiento, tanto de quien narra como de quien se narra, hay posibilidad de reconocimiento del otro y de sí mismo, porque existe la posibilidad de narrarme y contarme. Igualmente, tener la capacidad de narrar y narrarme ofrece la oportunidad de testimoniar y convertirme en testigo, es decir ser confiable ante un hecho, ser capaz de constatarlo"(1:71). Este elemento narrativo puede ser una herramienta conveniente, debido a que tanto el bioeticista como cualquier profesional de la salud, e incluso los pacientes, pueden narrar y narrar$\operatorname{se}(2)$.

Como bien dicen Carrió et al.(3), la medicina narrativa es un movimiento liderado por médicos que pretenden revisar sus modelos profesionales, tomando en cuenta tanto su práctica asistencial como sus propias experiencias como pacientes. La introducción de relatos en la formación médica, añaden, pone en cuestión el modelo biomédico tradicional, al valorar tanto el conocimiento subjetivo como el objetivo, el razonamiento inductivo como el deductivo, y la experiencia humana y la emoción tanto como la información científica. Es así que la medicina narrativa está abriendo un nuevo enfoque de renovación de la medicina que amerita ser explorado.

El paciente puede ser entendido como un texto, como un libro abierto, del que el médico puede y necesita mucho aprender. Es por eso que ahondaremos más adelante en la perspectiva narrativa de la comprensión del paciente y de su situación. Ello nos ayudará a descubrir cómo el paciente es objeto de la compasión de los médicos, pero también cómo se convierte en sujeto de esa compasión. Para una mayor comprensión del enfoque que se le ha dado a la compasión en el presente trabajo recomendamos revisar un estudio que desarrolla el tema con mayor profundidad(4). Debe definirse qué es, cómo se inicia la medicina narrativa y de dónde surge la compasión, con el fin de ubicar mejor al lector en la intencionalidad de la propuesta.

El objetivo de este trabajo consiste en evidenciar que una de las mejores maneras de aproximarse a un paciente es a través de la compasión. Pretendemos mostrar que la actitud compasiva se construye progresivamente, mediante el cultivo personal de otras virtudes o actitudes fundamentales para aproximarse al paciente, y hacemos algunas propuestas que pueden ser incluidas en la formación básica del profesional de la salud.

\section{El paciente como "texto"}

En reflexiones anteriores hemos hablado del paciente como texto - como un libro abierto- y sus implicaciones en la bioética(5). Como dice Villarroel, apoyado en Ricouer, "el hecho de que el paciente sea visto como un texto es sinónimo de que en su ser de enfermo pueden emerger palabras que muchas veces deben ser extraídas del silencio, palabras cuyo decir es siempre múltiple y están a la espera de nuevas interpretaciones que decidan su significación, palabras que, además, estarán abiertas a los muchos que puedan leerlas, a todas las instancias sociales que pueden proveer salud, en mayor o menor medida"(6:44-45).

Para comprender mejor al paciente, para leerlo en profundidad, se requiere la actitud compasiva. En la ausencia de compasión los pacientes están descontentos y los profesionales lamentan una pérdida de significado y gratificación en su 
trabajo(1). La compasión, como dice Post, es una cualidad esencial en el óptimo cuidado médico y constituye una sabiduría perenne y universal en la ética médica. Por ello, la compasión puede manifestarse como movimiento del corazón(4), que se construye poco a poco mediante un proceso de sensibilización y se da a través de experiencias tanto negativas como positivas, es decir, tanto en el dolor como el sufrimiento de un paciente, en su mejoría o alegría por el éxito de un tratamiento o cura de una enfermedad. La compasión, vista de esta manera, abarca toda la realidad del paciente y, de acuerdo con la analogía que sugerimos, conduciría a una comprensión más completa del "texto".

La compasión implica desarrollar la capacidad de asombro, como apertura a la realidad y como parte esencial de esa realidad, los rostros concretos de las personas que sufren o pasan por alguna necesidad(4). Es esa misma capacidad de asombro la que se requiere cuando se desea gozar de la lectura de un libro, de cualquier texto; de lo contrario será una experiencia amarga y los contenidos no serán bien comprendidos.

A continuación, describimos algunas actitudes que, de no existir, es muy difícil que se llegue a ser verdaderamente compasivo.

\section{Humildad}

La lectura de un libro está dada por un sinnúmero de motivaciones. Sin detenernos en particular en ellas, se puede afirmar que siempre se busca que el libro nos diga algo nuevo, algo que no sabemos, algo visto desde otra perspectiva. En el fondo, dejamos que el libro sea lo que es y, al leerlo, nosotros nos enriqueceremos con su lectura. Es así que, si un libro dijera todo lo que ya sé, que no me aporta nada nuevo, y dice lo que yo quiero que diga, probablemente no lo leeríamos. Pues bien, esa actitud con la que nos aproximamos a un libro dejando que el libro sea lo que es, es la humildad. Esto se da porque en el fondo nos dejamos asombrar por lo que nos propone el libro.

El asombro presupone una actitud humilde, y la compasión verdadera no se da sin asombro:

“El sentimiento de admiración que está en el inicio de toda reflexión sapiencial solo es posible gracias a una actitud fundamental de humildad: el reconocimiento de la propia ignorancia y de los límites de la propia inteligencia. Es así como el ser humano queda radicalmente capacitado para avanzar en el camino que lo conduce al hallazgo de respuestas cada vez más fundantes, pues, gracias a la humildad, somos conscientes de la riqueza insondable de la realidad y, al mismo tiempo, de la limitada capacidad del entendimiento humano para abarcar y penetrar dicha realidad en toda su complejidad y amplitud. La persona humilde sabe que detrás de cada interrogante resuelto late una nueva pregunta que le incita a avanzar respetuosa y atenta por los senderos que cosas, hechos y personas le señalan. El humilde es, por consiguiente, sumamente dócil, esto es, habitualmente dispuesto a dejarse enseñar por la realidad y por los demás" (7:5).

Además, la compasión, como es descrita por Guerrero e Izuzquiza(8), es silenciosa y humilde; a menos que la compasión vaya acompañada de humildad, dice Lachmanova(9), no es verdadera compasión. Es por eso que "si un corazón es regido por el orgullo, mostrará una falta de compasión para con los demás"(9:23). La verdadera actitud compasiva es la que se deja enseñar por la realidad. La verdadera persona compasiva es la que ve en el paciente un texto que tiene todo por enseñarle, que tiene un número de páginas que esperan ser leídas.

Quien lee un libro, quien lee un texto, se olvida de cierta manera de sí mismo, para poder comprender lo que el texto le dice. La compasión implica un olvido del yo. Pero no es desentenderse de uno mismo, es un olvido pasajero. Dice García-Baró: "la compasión verdadera, la que es compasión a secas y no compasión de, necesita de un olvido pasajero del Yo, de la memoria, de aquello que nos hace diferentes los unos de los otros. Cuando no somos memoria, sino puro presente estamos en disposición de acoger al Tú como otro Yo y no como un objeto. Podemos entonces hacer nuestras sus preocupaciones. Pero estos momentos de auténtica compasión son realmente escasos para nuestra existencia"(10:214). Siendo humildes podemos hacer nuestras las preocupaciones de los demás. La soberbia, que es todo lo opuesto a la humildad, nos impide esa apertura al otro y 
a sus necesidades, pues, como dice Polaino, "la persona soberbia sólo está pendiente de poner de manifiesto su excelencia personal o lo que ella considera le hace ser excelente (...) Pero no está atenta a los bienes ajenos, de los que casi nunca se alegra, sino que trata de minimizarlos o quitarles la importancia que tienen. Se muestra más bien partidaria del espíritu justiciero que devuelve mal por mal, porque en su corazón no hay lugar para el perdón, la comprensión o la tolerancia”(11).

\section{Soledad}

Es usual que, por las distintas ocupaciones y el ritmo de vida actual, la mayoría de las personas que viven en una ciudad hagan dos cosas al mismo tiempo: por ejemplo, hablar por teléfono y limpiar el escritorio, o salir a trotar y escuchar música, entre muchos otros ejemplos. No obstante, cuando nos abocamos a la tarea de leer, no es posible hacer otra cosa al mismo tiempo, y si la hacemos suele quedar mal hecha. ¿Por qué? Porque necesitamos la concentración para leer. Quien decide leer un libro, una revista o un texto en general necesita de un espacio vital que le proporcione un cierto grado de soledad para hacerlo. Análogamente con el paciente, al que consideramos como un texto, con el fin de proporcionarle la atención debida, no podemos llegar haciendo otras cosas, hablando por teléfono, enviando mensajes de "chat" por el celular, ni buscando arreglar aquel aparato que se nos descompuso.

Pues bien, la verdadera actitud compasiva requiere de la soledad, como dice Nouwen: "La compasión es el fruto de la soledad. (...) la purificación y transformación que tienen lugar en la soledad se manifiestan en la compasión"(12:26). Es en esta soledad en la que se gesta la verdadera compasión. Cuántas veces los médicos creen que entre más pacientes atiendan, más van a ser compasivos. Es cierto, el contacto con los pacientes los va educando en esas actitudes necesarias para el trato con ellos, pero son pocos los momentos que se dedican para estar solos, para poder digerir tantas experiencias que viven ellos mismos en el contacto con sus pacientes. A los médicos de hoy les falta algo de soledad. Y eso se ve reflejado en la poca soledad del encuentro médico-paciente pues, si bien es un encuentro - como dijimos-, requiere de soledad. Si el médico no busca que su encuentro con el paciente esté teñido por esa sana soledad, no explotará la riqueza de dicho encuentro, quizá no resuelva todos los problemas que tiene que resolver y no salga enriquecido por lo que ese encuentro le puede aportar. Pensamos que la falta de soledad para ser compasivo no es negociable. Se podrá ser un buen autómata de la medicina, pero no una persona que trate a sus pacientes como personas. En este sentido, Nouwen nos ilumina de la siguiente manera:

"Esta solidaridad compasiva crece en la soledad. En la soledad nos percatamos de que nada humano nos resulta ajeno, de que las raíces de todo conflicto, guerra, injusticia, crueldad, odio, celos y envidias están profundamente ancladas en nuestros corazones. En la soledad nuestro corazón de piedra puede ser cambiado en un corazón de carne, un corazón rebelde en corazón contrito, y un corazón cerrado en un corazón capaz de abrirse a todos los que sufren, en un gesto de solidaridad"(12:27).

$\mathrm{Y}$ es que no cabe duda de que ser compasivo es difícil. Tenemos que partir de allí. Quien crea que ser compasivo es simple empatía, sonreír al paciente cada vez que lo visita y brindarle un trato amable, está equivocado. La compasión incluye todo esto, pero va mucho más allá. Por eso dice Nouwen: "No subestimemos lo mucho que cuesta ser compasivo. La compasión es difícil porque requiere disposición interior a ir con otros al lugar en que ellos son débiles, vulnerables, aislados y rotos" (12:26).

\section{Aprender a estar}

¡Cuántas veces escuchamos entre esposos, amigos, novios, hermanos de comunidad, que reclaman que muchas veces sus compañeros los escuchan, pero simplemente no están ahí! En el mundo de hoy es frecuente escuchar que son pocos los que escuchan de verdad al otro, y dentro de los que escuchan, muchos lo hacen, pero pensando en otras cosas, en sus ocupaciones, en sus problemas, etc. Pues bien, para ser compasivo hay que aprender a "estar".

Quien no es capaz de desconectarse de internet, de su acelerado ritmo de vida, de las presiones del trabajo, del estudio —o simplemente no puede 
estar en silencio-, no podrá tomar un libro y sentarse a leer. Por el contrario, quien logra desconectarse y apartarse un poco de todas esas preocupaciones, consigue sacar el tiempo para tomar un libro y leerlo. Un buen lector aprende a estar con el libro; un buen lector sabe que vale la pena dedicarle tiempo; un buen lector busca una postura cómoda para degustar la lectura. Una postura incómoda conducirá al lector a abandonar la lectura del texto. Sencillamente, un buen lector aprende a estar.

Para que la compasión sea verdadera compasión, el médico debe aprender a estar con su paciente, no solo dedicarle el tiempo suficiente para poder ir leyendo su situación, sino que se sienta cómodo para una buena lectura, de la misma manera que alguien se acomoda para leer un libro. Es cierto que una consulta no podrá muchas veces asemejarse a esa lectura, pero sí requerirá el nivel de concentración, atención y sensibilidad para realizar una buena lectura de la situación del paciente. Por ejemplo, cuando éste busca ser escuchado por su médico, pero este último no toma una postura para escuchar, es probable que el paciente se desanime y no narre todo lo que le sucede, porque pareciera que lo que está diciendo incomoda al médico, pues éste no se ha tomado el trabajo de tomar una postura de escucha, sencillamente no está como debería estar ante el paciente.

Dice García-Baró: "La verdadera compasión sólo es posible en la palabra básica Yo-Tú, en el modo de la relación. Necesita, por tanto, cumplir una serie muy estricta de requisitos: debe darse en una dimensión temporal de puro presente, no puede ser buscada, pues requiere de la pasividad del sujeto y no puede mediar en ella ningún signo ni uso de razón" (10:215). Aprendemos a estar cuando valoramos el presente, cuando valoramos que el encuentro con tal o cual persona es único, que no sabemos si se repetirá; quizá, si fuéramos conscientes de que nuestros encuentros con personas concretas podrían no repetirse, nuestra actitud en dicho encuentro sería muy diferente y, si afortunadamente continúan dichos encuentros, aprenderemos a estar cada día un poco más.

\section{Dejar ser}

El paciente no puede ser víctima de juicios teme- rarios, como podría suceder con un texto cualquiera. Desde el momento en que un libro es catalogado como "aburrido", "pasado de moda", su imagen se ve mancillada de alguna manera. Es necesario dejar que estos sean simples comentarios, que no afecten el juicio de los que no lo han leído o que lleven a que la persona se cierre a todo lo que podría aportarle en su conjunto el contenido del texto. "Para estar al servicio de otros, dice Nouwen, tenemos que renunciar a medir el sentido y valor de nuestra vida con el metro ajeno. (...) dejar de juzgarlos, dejar de evaluarlos y liberarnos de este modo para poder ser compasivos. La compasión nunca puede coexistir con el juicio, porque el juicio crea la distancia, la distinción que nos impide estar realmente con el otro" (12:27).

Cabe aclarar que, ante un paciente, un médico o profesional de la salud debe emitir juicios; el problema está cuando se emiten juicios temerarios y se cataloga a los pacientes como mercancía. Es por eso que Nouwen afirma que quien busca en todo momento juzgar a otra persona lo único que hace es distanciarse de ella, pues los juicios endurecen el corazón y así lo único que se genera es una mayor distancia entre el médico y el paciente, y se hace difícil la vivencia de la actitud compasiva. Los juicios nos llevan a encasillar a las personas, a no darles respiro, a etiquetarlas y quedarnos con una imagen de ellas que quizá no es la que mejor las refleja. En fin, lo problemático es no dejar ser al paciente que sea lo que es, reducirlo a una simple etiqueta, ya de la habitación que ocupa, el número asignado en la sala de cuidados intensivos o su patología.

Dice Post(1) que la narrativa de la enfermedad debe ser respetada, que ningún paciente puede ser reducido a un acertijo biológico que debe ser resuelto. Esta visión empobrecida del paciente, sumada al posible etiquetaje que se pueda hacer de ellos, puede conducir al médico a verse limitado por sus propios juicios, pues son estos límites autoimpuestos los que impiden estar disponibles para la gente y achican la compasión(12).

\section{La compasión es bidireccional}

Diríamos que hemos llegado al punto más importante de la comprensión del paciente como texto y sus implicaciones en la compasión. Pues el pa- 
ciente como todo texto, tiene mucho que aportar al médico, que es su lector. Es momento de recordar al médico y a los profesionales de la salud que vean al paciente no solo como a aquel a quien dan atención, curan sus enfermedades o dan cuidados paliativos, sino también como aquel de quien reciben. Siempre será más fácil dar que recibir. Por ello, siempre será más fácil ver al paciente como necesitado de compasión, sin que el médico se descubra como un lector que, durante toda la lectura de un texto, se mantiene en constante actitud de escucha y aprendizaje no solo de contenidos, sino de emociones y experiencias de vida humana que le proporciona el encuentro con el paciente. Ser compasivo puede ser más fácil que dejarse compadecer, como dice Lachmanova: "Nos resulta fácil comprender que saber dar constituye una de las dimensiones de la compasión. Saber recibir es otra dimensión, de tal modo que respetemos la dignidad del otro"(9:73).

El problema ha estado en la poca comprensión de lo que es la enfermedad. Se ha visto siempre como algo físico, pero va mucho más allá, ya que la enfermedad, aunque es una ausencia de salud, no es un estado en el que se deja de ser algo, una carencia, una ausencia de identidad(13). A veces pareciera que los médicos se aproximaran a los pacientes como si hubiera que erradicar por completo la enfermedad, pues no erradicarla puede ser considerado a veces como un fracaso. Pero el problema no es que haya enfermos, dice Imíz$\operatorname{coz}(13)$, sino que haya sanos que no conozcan su posibilidad de enfermar, o que haya especialistas que estén convencidos - o nos convenzan- de la posibilidad de erradicar la enfermedad o los estados enfermales del hombre; es decir, que el estado de salud nos dé unas convicciones erróneas sobre nosotros mismos que nos hagan pensar que la enfermedad es un retraso, una falta de conocimientos o un fallo del sistema.

En clínicas y hospitales, a veces se percibe que el paciente es menos que todos los que trabajan allí -médicos, enfermeras, personal administrativo, etc. - y es que "si los demás se convierten en meros objetos de mi cuidado y generosidad, si no les doy la oportunidad de que también me den algo, y sobre todo si no les hago saber que son un don en mi vida, les estoy degradando"(9:74). Peor aún, no solamente se les degrada, sino que, al final, el pobre, el necesitado o el enfermo no quieren salir de donde están(9).

Por otro lado, pensar que la relación médico-paciente es unidireccional no solo tiene consecuencias de instrumentalización del paciente, sino que se da una "instrumentalización mutua"(8:69). Esto es precisamente lo que sucede muchas veces con los médicos. Es muy conocido el dicho de que "los médicos no son buenos pacientes" y de cierta forma este dicho refleja esa dificultad que tienen de recibir en general, de recibir ayuda. Pero si no desarrollamos esa disposición para recibir es difícil que podamos ser compasivos. $\mathrm{Al}$ recibir se da un proceso de sensibilización y de ablandamiento del corazón que nos posibilita para que, con esa misma sensibilidad, podamos servir y salir al encuentro especialmente de los más débiles.

\section{Competencias narrativas para el encuentro médico-paciente}

De acuerdo con lo que hemos mencionado en estudios anteriores(4), podemos decir que no se requiere estar ante situaciones de dolor o sufrimiento para desarrollar un corazón compasivo. Lo cual nos lleva a pensar que los médicos y los estudiantes de medicina pueden disponer y entrenar su corazón para ser compasivos, no solo en la atención a pacientes en clínica u hospital, sino que se puede aprovechar otras instancias de la vida diaria para crecer en dicha actitud compasiva. Por ejemplo, los doctores que pasan por un proceso de "burn out" muestran evidencias de mejoría cuando van en viajes de misiones médicas de periodos cortos y se reconectan con su deseo principal de cuidar a otros(1). Campbell(1) afirma que los viajes de misiones médicas de dos semanas a Sudamérica contribuyeron a bajar los puntajes en las escales de "burn out" a su retorno; dichos puntajes continuaron mejorando en los seis meses siguientes.

Nos concentraremos ahora en la escritura de relatos, como una manera de adquirir un corazón más compasivo. El simple hecho de narrar las experiencias vividas contribuye a un ablandamiento del corazón, piedra angular de la actitud compasiva, así como el crecimiento de la capacidad de asombro, estrechamente ligada a ésta.

La doctora Rita Charon propuso la medicina na- 
rrativa como un modelo para una práctica médica humana y efectiva, pues ésta requiere una competencia narrativa, es decir, la habilidad para reconocer, absorber, interpretar y actuar en las historias y condiciones de los demás(14). La adopción de métodos como la lectura de literatura y escritos reflexivos, afirma Charon(14), permite a la medicina narrativa examinar e iluminar cuatro de las situaciones centrales de la medicina: médico y paciente, médico consigo mismo, médico y colegas, y médicos y sociedad. Con esta competencia narrativa los médicos pueden alcanzar y unirse a sus pacientes en la enfermedad, identificar sus propios recorridos en la medicina, reconocer su cercanía y responsabilidades con otros profesionales de la salud, e inaugurar un discurso consecuente con el público en general sobre el cuidado de la salud. Los médicos aprenden de las situaciones de los pacientes de muchas maneras, incluyendo las narraciones y las escritas. Los médicos registran los estados de ánimo, los silencios, y los cambios corporales de los pacientes; de esta manera, van absorbiendo, sin necesidad del uso de las palabras, un conocimiento acerca de ellos(15).

Pero, ¿qué utilidad real puede tener para un médico - dedicado a los asuntos asistenciales, docentes o investigadores de su especialidad- una selección de novelas, poemas o piezas dramáticas?, se pregunta Navarro(16). Pues este es uno de los retos a los que se ha visto abocada la medicina narrativa. Charon(17) afirma que, como base de la cultura, la religión, la familia y la comunidad, la narración es una vía mediante la cual somos más nosotros mismos, nos permite conocer nuestro ser. En el caso de la medicina, que es el que aquí nos atañe, se han dado grandes avances; ya para el 2007 los currículos y proyectos narrativos se habían difundido en los Estados Unidos, Canadá, Europa, Gran Bretaña, Latinoamérica, Medio Oriente y Australia(15).

Con el fin de que los médicos y estudiantes de medicina puedan adquirir, fortalecer y explotar sus capacidades narrativas, queremos mencionar dos importantes iniciativas desarrolladas recientemente. En primer lugar, la lectura de otros textos distintos a los de medicina pues, como dice Navarro: "En esta búsqueda, los libros de texto habrán de resultarle de escasa utilidad. Cierto es que los cuadros sintomáticos, los signos clínicos, los datos analíticos y los resultados de las más modernas técnicas diagnósticas vienen recogidos con extraordinaria minuciosidad en estos sesudos tratados; pero rara vez ocurre igual con los sentimientos o las sensaciones más íntimas del paciente. $\mathrm{Si}$ es esto lo que buscamos, más vale volver la vista a los textos escritos no por los más eminentes discípulos de Hipócrates, sino por quienes mejor han sabido observar, interpretar y expresar los entresijos del alma humana: los grandes escritores de la literatura universal" (16:98). Es así que Navarro(16) ha hecho una lista de 70 textos que debería incluir el médico en su formación, por ejemplo, Middlemarch, de George Eliot, El médico imaginario, de Molière, y El doctor inverosímil, de Ramón Gómez de la Serna, entre otros.

Además de la lectura de obras literarias, se ha comprobado la importancia de las narraciones escritas; así, la revista Medicina Narrativa, de la Pontificia Universidad Javeriana, que en cuatro años de edición (2011 a 2014) ha publicado 353 artículos, escritos por estudiantes y profesores. De estos artículos, la mayor cantidad ha estado en cuatro áreas temáticas, marcadas así por la revista: 19,0\% (66 artículos) acerca de la "vocación médica"; 17,6\% (62 artículos) sobre "prosa diversa"; $16,1 \%$ (57 artículos) relativa a "relatos de enfermedad" y 11\% (39 artículos) "sobre la muerte" y en particular de la muerte de seres queridos. Por otro lado, habiendo comenzado con 24 artículos en el primer número de la revista de 2011, en los dos números de 2014 se publicaron 60 y 56 artículos. De acuerdo con lo anterior, vemos el creciente interés, tanto de profesores como de estudiantes, de narrar sus experiencias médicas de diversas maneras: en poesía, relatos en prosa, cuentos, etc. Incluso, a partir del 2013, se creó una nueva sección en la revista — "Facultad en pañales" - , en la que se publica fotos y pequeñas biografías de los narradores. Existe por tanto un fuerte deseo de contar la vida, de narrar y narrarse, pues, como dice la doctora Russo:

"La literatura, así como la filosofía, puede ser un instrumento precioso para que se formen generaciones de médicos sensibles a la historia del dolor del paciente, además de a la mera historia clínica.

\footnotetext{
${ }^{2}$ Datos tomados por el autor luego de una revisión de cada uno de los números de la revista publicados en el sitio web http://revistas. javerianacali.edu.co/index.php/medicinanarrativa
} 
Si sólo la persona humana tiene historia, más precisamente biografía, porque a través de su libertad madura en una continuidad de acciones, no puede haber cuidado de la persona sin la atención a su historia, es decir, sin el respeto de su libertad"(18:131).

La habilidad de narrar y de representar lo que observamos o sufrimos en momentos de enfermedad nos da acceso a un conocimiento que, sin la representación narrativa, quedaría fuera de nuestras conciencias(19); es por ello que, a pesar de recibir resistencia a introducir este tipo de enfoque en la formación médica, es importante tenerla muy en cuenta. Sus resultados se verán más adelante, cuando haya generaciones de médicos formados bajo esta escuela de la medicina narrativa.

\section{Conclusión}

Estas reflexiones nos parecen lo suficientemente paradigmáticas para señalar que el paciente puede ser considerado con "texto", en cuanto es un libro abierto que ofrece gran cantidad de información, tan vasta y tan compleja como puede haber sido su vida, y no solo sus episodios de enfermedad, dolor o sufrimiento. En esta instancia, el paciente sigue siendo objeto de estudio del médico, el cual busca ayudarle en su proceso de curación de la enfermedad —aliviando sus dolores_ y en su recuperación.
Asimismo, entender como un texto al paciente permite descubrir ciertas actitudes que son imprescindibles para hacer una lectura a fondo sobre él, las cuales se constituyen en las piezas con las que constituye una verdadera actitud compasiva hacia el paciente. Queda claro que esta actitud no es una pérdida de tiempo para el médico ni un desgaste emocional, sino que constituye una fortaleza para crecer en la efectividad de su ejercicio profesional, en sus relaciones con otros médicos y demás profesionales de la salud, así como en el proceso formativo de los estudiantes de medicina.

Como libro abierto, el paciente reclama la categoría de sujeto, de un individuo con el que el médico puede no solo tener un verdadero encuentro personal, sino que también puede y debe aprender mucho de él. Para ello, es importante que los médicos dediquen tiempo en su formación médica inicial, así como en su formación permanente, a explorar y explotar el valor de las narraciones, para instruirse a través de la lectura de libros y obras de literatura en general, o de la escritura de textos que les ayuden a categorizar sus experiencias vividas y las de los pacientes. La acogida de estas iniciativas enfocadas en la narrativa es muy alta, pero queda por continuar indagando, si sus resultados contribuyen a la tan anhelada humanización de la medicina. 


\section{Referencias}

1. Post S. Compassionate care enhancement: benefits and outcomes. The International Journal of Person Centered Medicine 2011; 1(4): 808-813.

2. Alzate NA. Aportes de la hermenéutica ricoeuriana a la bioética. [Tesis]. Bogotá: Pontificia Universidad Javeriana/Instituto de Bioética; 2011.

3. Carrió S, De Cunto C, Cachiarelli N, Ceriani C, Catsicaris C, Usandivaras I. Medicina narrativa en pediatría: relato de una experiencia. Arch Argent Pediatr 2008; 106(2): 138-142.

4. Rosas CA. Humanización de la medicina: lástima o compasión. Academia (Número Especial): 128-142.

5. Rosas CA. El paciente como “texto” según Ricoeur: implicaciones en bioética. Bioética 2014; 22(2): $234-240$.

6. Villarroel R. Bioética hermenéutica. Acta Bioethica 2000; 6: 143-57.

7. Irízar LB. En busca de nosotros mismos. Acerca de la necesidad de la sabiduría para el hombre de hoy. Civilizar. Ciencias Sociales y Humanas 2005; 9:1-18.

8. Guerrero JA, Izuzquiza D. Vidas que sobran. Los excluidos de un mundo en quiebra. Santander: Sal Terrae; 2003.

9. Lachmanova K. Compasión. Salamanca: Ediciones Sígueme; 2005.

10. García-Baró M. y Villar A, (eds.). Pensar la compasión. Una reflexión sobre la compasión a partir de la filosofía de Martin Buber. Madrid: Universidad Pontificia de Comillas; 2008: 211-215.

11. Polaino-Llorente, A. En busca de la autoestima perdida. Bilbao: Desclée de Brouwer; 2004.

12. Nouwen H. El camino del corazón. Buenos Aires: Editorial Guadalupe; 2005.

13. Imízcoz T. Contarse y curarse. Reflexiones sobre literatura y enfermedad. In: Anrubia E, (ed.). La fragilidad de los hombres. Madrid: Fuenlabrada; 2008: 103-24.

14. Charon R. Narrative Medicine: A Model for Empathy, Reflection, Profession, and Trust. JAMA 2001; 286(15): $1897-$ 1902 .

15. Charon R. What to do with stories. The sciences of narrative medicine. Canadian Family Physician 2007; 53(August): 1265-1267.

16. Navarro F. Biblioteca literaria para médicos I. Med Cine 2015; 11(2): 97-104.

17. Charon R. At the Membranes of Care: Stories in Narrative Medicine. Acad Med 2012 March; 87(3): 342-347.

18. Russo M. Bioética y literatura. Persona y Bioética 2007; 2(27): 121-131.

19. Charon R. "Illuminating the Real of Health, Expressing the Real of Illness" - For the Narrative and Medicine: Caring for the Future Conference (Lisbon, Calouste Gulbenkian Foundation and Faculty of Social and Human Sciences, 5-7 March 2015). Disponible en: http://goo.gl/z7oIGZ (March 12th , 2015).

Recibido: 13 de noviembre de 2015

Aceptado: 7 de junio de 2016 